\title{
Synthesis of Shaped Beam for Base-station Antennas in Cellular Mobile Communications
}

\author{
Ruying Sun \\ School of Informatics, Linyi Normal University, Linyi 276005, China \\ E-mail: srysd@163.com
}

\begin{abstract}
Genetic Algorithms is used to synthesize shaped beam and optimize base-station antenna coverage in the article. Two cases have been studied: one is to change the feeding amplitude and phase of each element; the other is to change the feeding phase of each element and the distances between them. And then we combine the Genetic Algorithms with FEKO to synthesize antenna patterns. Comparing to the Woodward method, the results achieved by Genetic Algorithms are better than Woodward method because it is nearly to the ideal square-cosecant patterns.
\end{abstract}

Keywords: Shaped Beam, Antenna Synthesis, Base-station Antennas, Genetic Algorithms, FEKO 4.0

\section{Introduction}

The object functions for synthesis of array antennas usually have the characteristics of multi-parameters, non-differentiable even discontinuities. The optimization of pattern function is non-linear problem. Traditional optimization techniques search for the best solutions, using gradients or random searching. Gradient methods are efficient, but have disadvantages of getting stuck in local minima, requiring gradients calculations, working only on continuous parameters. Random-search methods don't require gradient calculations, but tend to be slow, and susceptible to getting stuck in local minima. Genetic-algorithm optimizer is robust global search method. Its research is based on probability, having advantage of avoiding getting stuck in local minima (Holland J.H, 1992, chapter3).

In mobile communications system, the radiation pattern of base-station antenna should have weak interference to adjacent areas, and generate intense and uniform radiation Intensity in cellular area (Rongcang HAN, 2006, 34). According to the system request, a particular type of elevation pattern is achieved by technology of beam forming

\section{Methods}

Here, there is a quick overview of genetic algorithms, much more detail on genetic algorithms is found in reference (Randy L, Haupt, 1994, 995).Genes are the basic building blocks of genetic algorithms. A gene is binary encoding of a parameter. In computer algorithm, a chromosome is an array of genes, a number of chromosomes make up one population. Each chromosome has an associated fitness function, assigning a relative merit to that chromosome.

The algorithm begins with a large list of random chromosomes. Fitness functions are evaluated for each chromosome. The chromosomes are ranked from the most-fit to the least-fit, according to their respective fitness functions. Unacceptable chromosomes are discarded, leaving a superior species-subset of an original list, which is the process of selection. Genes that survive become parents, by crossing over some of their genetic material to produce two new offspring. The parents reproduce enough to offset the discarded chromosomes. Thus, the total number of chromosomes remains constant after next iteration. Mutations cause small random changes in a chromosome. Fitness functions are evaluated for the offspring and mutated chromosome, and the process is repeated. The algorithm stops after a set number of iterations, or when an acceptable solution is obtained. Figure.1 is a flow chart of Genetic Algorithms (K.Markus, L.Vaskelainen., 1998, 461).

Suppose we consider an array of antenna elements uniformly spaced in a straight line along the $\mathrm{z}$ axis. The far-field radiation pattern produced by such an array may be expressed as

$$
E(\theta, \varphi)=\sum_{n=0}^{N-1} I_{n} E P_{n}(\theta, \varphi) e^{j\left(n k d \cos \theta+\beta_{n}\right)}
$$

where $I_{n}, \beta_{n}$ are the element excitation current amplitudes and phases, $E P_{n}(\theta, \varphi)$ are the individual array element patterns, $\mathrm{k}=2 \pi / \lambda$ is the free-space wave number, $\mathrm{d}$ is the separation distance between elements.

If the common assumption is made that all of the elements have identical element patterns, then equation (1) may be written in the form of

$$
E(\theta, \varphi)=E P(\theta, \varphi) A F(\theta)
$$




$$
A F(\theta)=\sum_{n=0}^{N-1} I_{n} e^{j\left(n k d \cos \theta+\beta_{n}\right)}
$$

Where $A F(\theta)$ is the associated array factor (Diógenes Marcano, and Filinto Durán, 2000, 14).

Now the main process of antenna synthesis in genetic algorithms is given below.

A. Establish decision variable and constraint condition, then encoding them.

$$
I_{n} \in[0,1], \beta_{n} \in[0,2 \pi], n=0,1,2, \cdots N-1
$$

B. Create optimization pattern:

$$
\min E_{m t}=\left[\frac{1}{Q} \sum_{i=1}^{Q}\left|e_{i}\right|^{2}\right]^{\frac{1}{2}}
$$

where

$$
e_{i}=\frac{T_{i}-F_{i}}{T_{i}}, i=1,2, \cdots, Q
$$

$T_{i}$ is the level of the desired radiation pattern at the point $\mathrm{Q}$, and $F_{i}$ is the level of the pattern generated by genetic algorithms.

C. Define fitness function:

$$
F_{m}=\frac{1}{1+E_{m t}^{\alpha}} \quad \alpha \in(0,1]
$$

D. Define object function according to the characteristics of desired radiation pattern.

\section{Numerical Examples}

Now, we define an object function according to the request of base-station antenna in order to achieve intense and uniform radiation intensity in cellular area. The object function is defined as equation 8 .

$$
F_{\circ}=\left\{\begin{array}{c}
\sec \theta, \theta \in\left[0^{\circ}, 90^{\circ}\right) \\
0, \quad \text { el se }
\end{array}\right.
$$

Here $N=8, d=\lambda / 2$, array elements are 8 half-wave dipole, which structure in WinFEKO surroundings is described in Figure 2. Combining GA with FEKO 4.0 can take into account both the radiation pattern of array elements and the mutual coupling among them when synthesizing antenna array patterns.

Figure 3 shows the result of base-station antenna pattern using Genetic Algorithms in solid curve and the result of Woodward in dashed curve. The major lobe of both array factor patterns direct ground. The radiation of electromagnetic energy is more intensive and uniform in array factor produced by genetic algorithms than Woodward.

\section{Conclusions}

Anyway, Genetic Algorithms is a good method to synthesis of shaped beam. The synthesis of shaped beam for the base-station array antenna with 8 elements is a good case. The result achieved by Genetic Algorithms is nearly to the ideal square-cosecant patterns, which is better than Woodward method. Genetic Algorithms also can be used to synthesize arrays with unequal distances between elements. It can be applied in other topics of antenna synthesis.

\section{References}

Diógenes Marcano, and Filinto Durán, (2000). Synthesis of Antenna Array Using Genetic Algorithms. IEEE, Antennas and Propagation Magazine, vol.42, no.3, pp12 20.

Holland J.H. (1992). Adaptation in Nature and Artificial Systems. MIT Press, chapter3.

K.Markus, L.Vaskelainen. (1998). Optimization of synthesized array excitations using array polynome complex root swapping and genetic algorithms. IEE Proc-Microw Antennas Propag, vol.145, no.6, pp460 464.

Randy L, Haupt. (1994). Arrays Using Genetic Algorithm. IEEE Transactions on Antennas and Propagation, vol.42, no.7, pp993 999.

Rongcang HAN. (2006). Synthesis of Array Antenna Based on Genetic Algorithms. Master's thesis of University of Electronic Science and Technology of China (UESTC), 34 


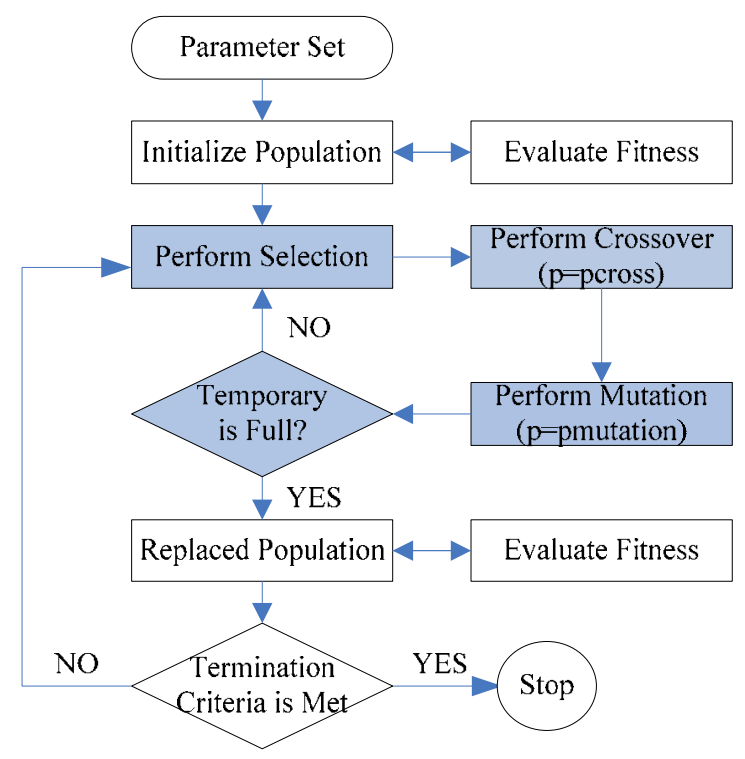

Figure 1. A flow chart of Genetic Algorithms

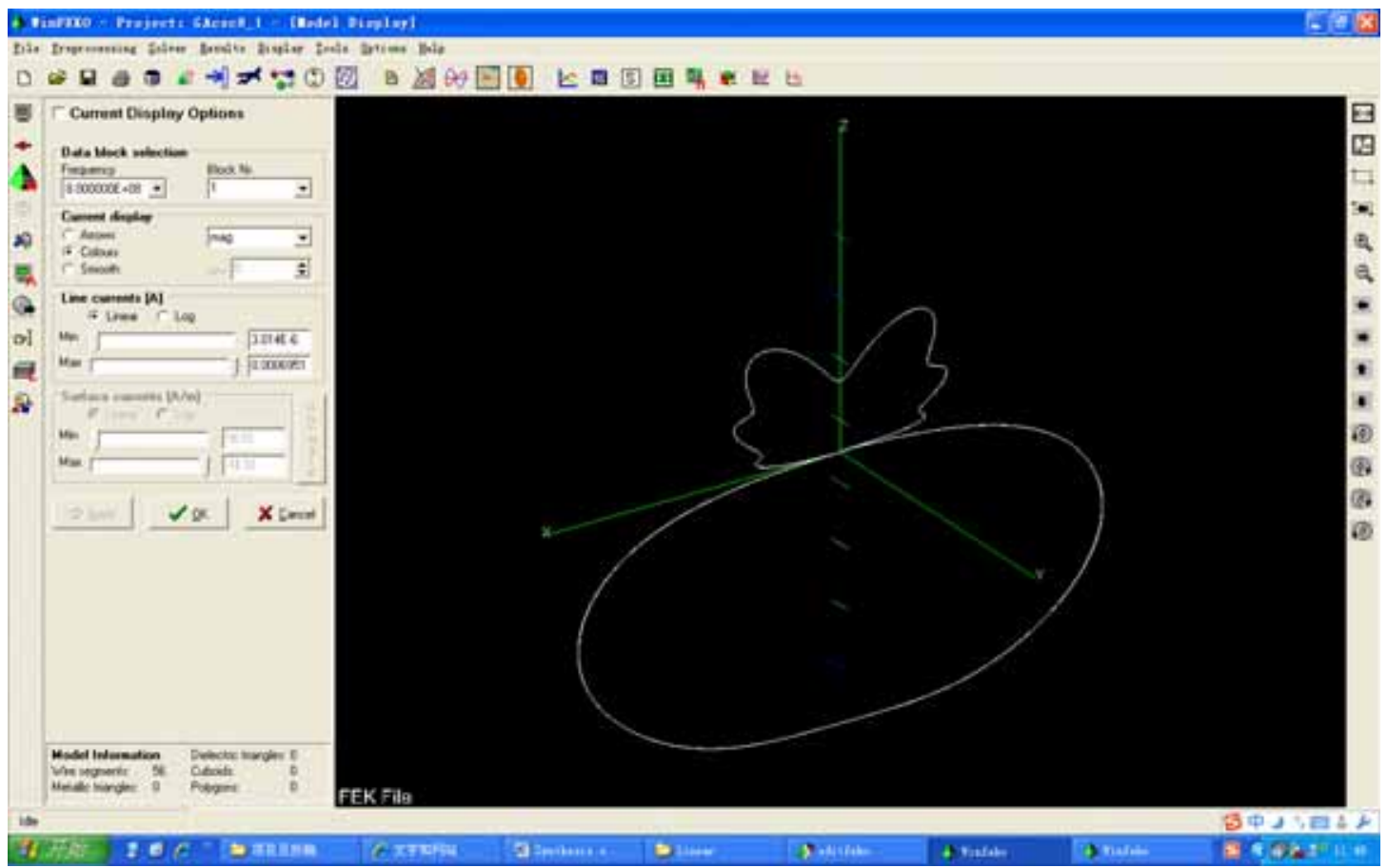

Figure 2. The structure of base-station antenna in WinFEKO surroundings 


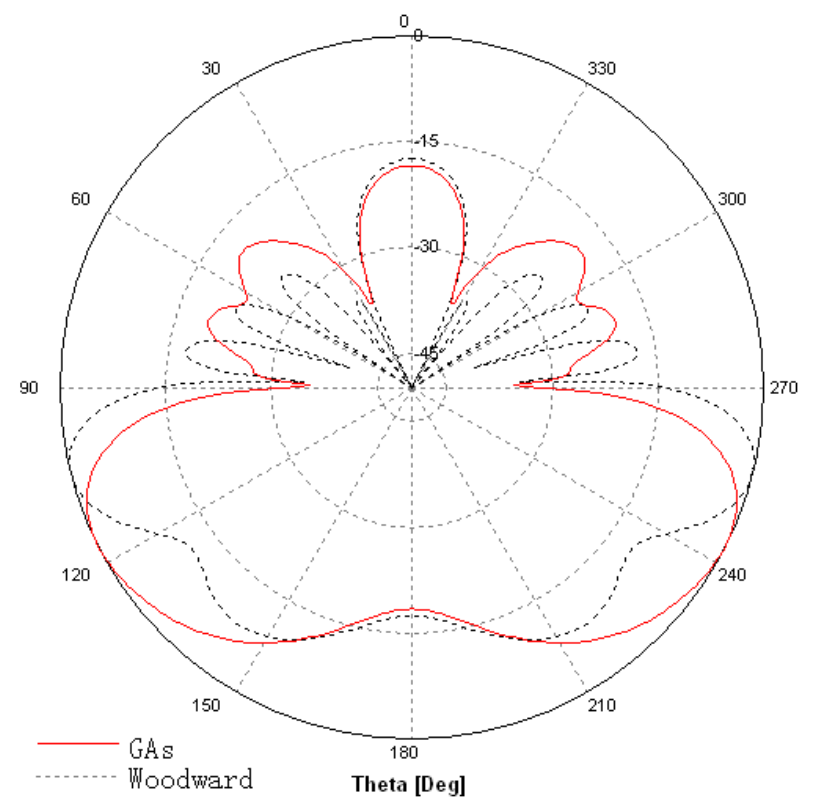

Figure 3. Shaped beam of base-station antenna generated by Genetic Algorithms and Woodward 